HUTMP B331

September 6, 2018

\title{
Supersymmetry and Fredholm Modules Over Quantized Spaces
}

\author{
David Borthwick*, Slawomir Klimek* ${ }^{* * 1}$, \\ Andrzej Lesniewski*2, and Maurizio Rinaldi*3 \\ *Lyman Laboratory of Physics \\ Harvard University \\ Cambridge, MA 02138, USA \\ ** Department of Mathematics \\ IUPUI \\ Indianapolis, IN 46205, USA
}

\begin{abstract}
The purpose of this paper is to apply the framework of non-commutative differential geometry to quantum deformations of a class of Kähler manifolds. For the examples of the Cartan domains of type I and flat space, we construct Fredholm modules over the quantized manifolds using the supercharges which arise in the quantization of supersymmetric generalizations of the manifolds. We compute an explicit formula for the Chern character on generators of the Toeplitz $\mathbb{C}^{*}$-algebra.
\end{abstract}

1 Supported in part by the National Science Foundation under grant DMS-9206936

2 Supported in part by the Department of Energy under grant DE-FG02-88ER25065

3 Supported in part by the Consiglio Nazionale delle Ricerche (CNR) 


\section{Introduction}

I.A. Since the early work on quantum mechanics ([15], [3], [12], [4]) by Heisenberg, Born, Jordan, and Dirac, it has been generally recognized that ordinary geometry does not apply to the subatomic world. In order to describe the physical phenomena in that world, the classical notion of phase space needs to be replaced by a non-commutative algebra of "quantum observables". The coordinates $p$ and $q$ on the phase space $\mathbb{R}^{2}$ are replaced by generators $p$ and $q$ that obey the famous commutation relation $[q, p]=i \hbar$. This "quantization" procedure amounts to studying a non-commutative deformation of a flat space, and, from a geometric viewpoint quantum mechanics emerges as some form of symplectic geometry on this non-commutative space. The classical algebra of functions on phase space arises as the $\hbar \rightarrow 0$ limit of the deformed algebra, and the Poisson bracket of two observables turns out to be the subleading term in the small $\hbar$ expansion of the commutator of the corresponding quantized observables. Much work has been done since the early quantum mechanics on extending this procedure to more general, non-flat phase spaces, resulting in powerful theories known as geometric quantization, deformation quantization, quantum groups, etc.

I.B. In the mid-eighties A. Connes [9] proposed a general scheme of non-commutative differential geometry which is ideally suited to describe the geometry of quantum theory. The central concept of this scheme is a non-commutative $\mathbb{C}^{*}$-algebra $\mathcal{A}$ which plays the role of an algebra of continuous functions on the (putative) non-commutative space. The de Rham cohomology of a smooth manifold is replaced by the cyclic cohomology of $\mathcal{A}$, while elements of the group $K_{0}(\mathcal{A})$ play the role of vector bundles over the non-commutative space. Another central concept of Connes' framework is that of a $p$-summable Fredholm module over $\mathcal{A}$, which replaces the classical notion of metric structure on a manifold. This is defined as a triple $(\mathcal{H}, \rho, Q)$, where $\mathcal{H}$ is a $\mathbb{Z}_{2}$-graded Hilbert space, $\rho$ is an action of $\mathcal{A}$ by bounded operators on $\mathcal{H}$, and $Q$ is a self-adjoint operator on $\mathcal{H}$ which is odd with respect to the $\mathbb{Z}_{2}$-grading (see Section II for a precise definition). In examples, the operator $Q$ is often a Dirac type operator. To a Fredholm module over $\mathcal{A}$, Connes associates a fundamental cocycle in cyclic cohomology, called the Chern character. 
I.C. A conceptual framework of quantization which fits the scheme of non-commuative differential geometry was proposed by Rieffel in [19]. This framework relies on the use of $\mathbb{C}^{*}$-algebras and precise operator norm estimates (rather than formal power series in Planck's constant), and we refer to it as non-perturbative deformation quantization. Examples of quantized spaces studied within this framework include quantized tori (see [20] for a review and references) and quantized flat spaces (see [8] for some recent results and references). In [17], [7], [5], and [6] we studied quantum deformations of a class of hermitian symmetric spaces and superspaces, namely the Cartan domains and superdomains. In each case, we constructed a family of $\mathbb{C}^{*}$-algebras of "quantized functions" and verified that these $\mathbb{C}^{*}$-algebras are indeed quantum deformations of the corresponding classical algebras of functions. In this paper and its sequel we study Fredholm modules over these algebras and the associated Chern characters.

I.D. As explained by Witten in his work on supersymmetry [21], it is natural to regard Dirac type operators as generators of supersymmetries ("supercharges") in certain physical systems involving bosons and fermions. This suggests that a natural way of constructing Fredholm modules over a quantized manifold is first to quantize a supersymmetric generalization of the manifold (see e.g. [2] for an introduction to the theory of supermanifolds), and then to take $Q$ to be a supercharge generating the supersymmetry. Following this idea, we construct Fredholm modules over the quantized type I Cartan domains and over quantized flat spaces. Our construction relies on [5], where we studied the relevant supersymmetric theories. We introduced there the notion of a super Toeplitz operator and the $\mathbb{C}^{*}$-algebra generated by such operators. The Fredholm modules we construct bear a certain resemblance to those constructed for the Toeplitz algebra over the circle in Section 4.2 of [11].

I.E. The paper is organized as follows. In Section II we briefly review Connes' formalism of non-commutative differential geometry. In Sections III and IV we study Fredholm modules over the quantized type I Cartan domains, and in Section V we study Fredholm modules over quantized flat spaces. 


\section{Fredholm Modules and Their Chern Characters}

II.A. In this section we briefly review the notion of a $p$-summable Fredholm module over a $\mathbb{C}^{*}$-algebra $\mathcal{A}$. From a physical point of view, the concept of a Fredholm module captures the essential features of a quantum supersymmetric system: the $\mathbb{C}^{*}$-algebra $\mathcal{A}$ is the algebra of observables, the Dirac operator is the supersymmetry generator, and its square is the Hamiltonian of the system.

Let $\mathcal{A}$ be a trivially $\mathbb{Z}_{2}$-graded $\mathbb{C}^{*}$-algebra (all elements are even). Recall ([9], [11]) that a $p$-summable Fredholm module over $\mathcal{A}$ is a triple $(\mathcal{H}, \rho, Q)$ such that:

(i) $\mathcal{H}$ is a $\mathbb{Z}_{2}$-graded Hilbert space. We denote by $\Gamma$ the grading operator and by $\mathcal{H}_{0}$ and $\mathcal{H}_{1}$ the homogeneous subspaces of $\mathcal{H}$.

(ii) $\rho: \mathcal{A} \longrightarrow \mathcal{L}(\mathcal{H})$ is a grading preserving $*$-homomorphism of $\mathcal{A}$ into the algebra of bounded linear operators on $\mathcal{H}$. For notational simplicity, we will suppress $\rho$ in all formulas throughout the rest of this paper.

(iii) $Q$ is a self-adjoint operator on $\mathcal{H}$ which is odd under the $\mathbb{Z}_{2}$-grading, i.e.

$$
Q \Gamma+\Gamma Q=0,
$$

and such that for any $\epsilon>0$,

$$
\left(Q^{2}+I\right)^{-1 / 2} \in I_{p+\epsilon}(\mathcal{H}) .
$$

Here, $I_{p}(\mathcal{H})$ denotes the $p$-th Schatten class of operators on $\mathcal{H}$. It is natural to regard the smallest number $p$ in (II.2) as the dimension of the non-commutative space.

(iv) The subalgebra $\mathcal{A}^{Q}$ consisting of those $a \in \mathcal{A}$ for which the commutator $[Q, a]$ is bounded is dense in $\mathcal{A}$.

We refer to $Q$ as the Dirac operator. In the following, we will denote $H:=Q^{2}$ and refer to it as the Laplace operator. Clearly, $H$ is a positive self-adjoint operator.

II.B. A $p$-summable Fredholm module defines a fundamental cocycle in the cyclic cohomology of $\mathcal{A}^{Q}$, called the Chern character [9]. In this paper we use the Chern character of [16], which is a cocycle in the entire cyclic cohomology [10] of $\mathcal{A}^{Q}$. It has the advantage of being expressed in terms of the heat kernel of the Laplace operator (very much like the McKean-Singer formula in index theory), which leads to useful integral representations. Its truncation to cyclic cohomology is discussed in [11]. This cocycle, $\mathrm{Ch}^{\beta}(Q)=\left\{\mathrm{Ch}_{2 k}^{\beta}(Q)\right\}_{k=0}^{\infty}$, where each $\mathrm{Ch}_{2 k}^{\beta}(Q)$ is a $(2 k+1)$-linear functional on $\mathcal{A}^{Q}$, is defined as follows. For $a \in \mathcal{L}(\mathcal{H})$ and $t>0$ we define the unbounded, densely defined operator

$$
a(t):=e^{-t H} a e^{t H} .
$$


For $\beta>0$ and $a_{0}, a_{1} \ldots, a_{2 k} \in \mathcal{A}^{Q}$ we set

$$
\mathrm{Ch}_{2 k}^{\beta}(Q)\left(a_{0}, a_{1}, \ldots, a_{2 k}\right):=\beta^{-k} \int_{\sigma_{2 k}^{\beta}} \operatorname{Str}\left\{a_{0}\left[Q, a_{1}\right]\left(t_{1}\right) \ldots\left[Q, a_{2 k}\right]\left(t_{2 k}\right) e^{-\beta H}\right\} d^{2 k} t,
$$

where $\sigma_{n}^{\beta}:=\left\{\left(t_{1}, \ldots, t_{n}\right) \in \mathbb{R}^{n}: 0 \leq t_{1} \leq \ldots \leq t_{n} \leq \beta\right\}$, and where Str denotes the supertrace,

$$
\operatorname{Str}(A):=\operatorname{Tr}(\Gamma A) .
$$

The key analytic input ensuring the existence of (II.4) is the following inequality [16]. For $s_{j} \geq 0, j=0, \ldots, n$, with $\sum_{j=0}^{n} s_{j}=\beta$, and $A_{0}, A_{1}, \ldots, A_{n} \in \mathcal{L}(\mathcal{H})$,

$$
\left|\operatorname{Tr}\left(A_{0} e^{-s_{0} H} A_{1} e^{-s_{1} H} \ldots A_{n} e^{-s_{n} H}\right)\right| \leq \prod_{j=0}^{n}\left\|A_{j}\right\| \operatorname{Tr}\left(e^{-\beta H}\right)
$$

(we use this estimate with $s_{0}=t_{1}, s_{1}=t_{2}-t_{1}, \ldots, s_{n}=\beta-t_{n}$ ). The inequality (II.6) is proven using Hölder's inequality and holds for any positive operator in place of $H$.

II.C. For the case of Fredholm modules arising from the quantized Kähler supermanifolds discussed later in this paper, we will have two Dirac operators $Q_{1}$ and $Q_{2}$ which generate an $N=2$ supersymmetry algebra, namely

$$
\begin{aligned}
& Q_{1}^{2}=Q_{2}^{2}=H, \\
& Q_{1} Q_{2}+Q_{2} Q_{1}=0 .
\end{aligned}
$$

These two operators will be essentially self-adjoint on some dense subspace $\mathcal{P} \subset \mathcal{H}$ and will have the structure:

$$
\begin{aligned}
& Q_{1}=d+d^{*}, \\
& Q_{2}=i\left(d-d^{*}\right),
\end{aligned}
$$

where $d$ is an operator such that $d^{2}=0, \operatorname{Dom}(d)=\mathcal{P}$. We define $\mathcal{A}^{1}:=\mathcal{A}^{Q_{1}} \cap \mathcal{A}^{Q_{2}}$. Here we make an important assumption that $\mathcal{A}^{1}$ be dense in $\mathcal{A}$ (this is the key element which limits the generality of the following theorem). This will clearly be the case for the examples we consider.

Theorem II.1. Let $\left(\mathcal{H}, \rho, Q_{1}\right)$ and $\left(\mathcal{H}, \rho, Q_{2}\right)$ be Fredholm modules over $\mathcal{A}$, with $Q_{1}$ and $Q_{2}$ as in (II.8). Then the corresponding Chern characters define the same cohomology class in the entire cyclic cohomology of $\mathcal{A}^{1}$.

Proof. The theorem is proven through a homotopy argument. We can set $\beta=1$ with no loss of generality. We form the family of operators

$$
Q(\lambda):=Q_{1} \cos \lambda+Q_{2} \sin \lambda=e^{i \lambda} d+e^{-i \lambda} d^{*}
$$


for $0 \leq \lambda<2 \pi$, which interpolates between $Q_{1}$ and $Q_{2}: Q(0)=Q_{1}, Q(\pi / 2)=Q_{2}$. Clearly, $Q(\lambda)$ is essentially self-adjoint on $\mathcal{P}$, and the commutator of $Q(\lambda)$ with any element of $\mathcal{A}^{1}$ is bounded. We proceed as in [13] to show that $\frac{d}{d \lambda} \operatorname{Ch}(Q(\lambda))$ exists and is equal to a coboundary, so that $\operatorname{Ch}\left(Q_{1}\right)$ and $\operatorname{Ch}\left(Q_{2}\right)$ are cohomologous. Note that the technical assumptions (i) and (ii) of Section III of [13] are not satisfied in our case and we need to make some changes in the argument.

Observe that the $Q(\lambda)$ obey the following algebra:

$$
[Q(\lambda), Q(\mu)]=2 \cos (\lambda-\mu) H
$$

In particular, $Q(\lambda)^{2}=H$, and so $H$ is the Laplace operator corresponding to $Q(\lambda)$ for any $\lambda$. On $\mathcal{P}$ we can take the derivative

$$
Q^{\prime}(\lambda):=\frac{d}{d \lambda} Q(\lambda)=-Q_{1} \sin \lambda+Q_{2} \cos \lambda=Q(\lambda+\pi / 2)
$$

and we thus see the $Q^{\prime}(\lambda)$ is also essentially self-adjoint on $\mathcal{P}$. Define $G^{\lambda}=\left\{G_{2 k-1}^{\lambda}\right\}_{k=1}^{\infty}$, where each $G_{2 k-1}^{\lambda}$ is a $2 k$-linear functional on $\mathcal{A}^{1}$ given by

$$
\begin{gathered}
G_{2 k-1}^{\lambda}\left(a_{0}, \ldots, a_{2 k-1}\right) \\
:=\sum_{l=0}^{2 k-1}(-1)^{l+1} \int_{\sigma_{2 k}^{1}} \operatorname{Str}\left\{a_{0}\left[Q(\lambda), a_{1}\right]\left(t_{1}\right) \ldots\left[Q(\lambda), a_{l}\right]\left(t_{l}\right) Q^{\prime}(\lambda)\left(t_{l+1}\right)\left[Q(\lambda), a_{l+1}\right]\left(t_{l+2}\right)\right. \\
\left.\ldots\left[Q(\lambda), a_{2 k-1}\right]\left(t_{2 k}\right) e^{-H}\right\} d^{2 k} t
\end{gathered}
$$

for $a_{0}, \ldots, a_{2 k-1} \in \mathcal{A}^{1}$. Observe that the heat kernels in $\operatorname{Ch}(Q(\lambda))$ are independent of $\lambda$ and so differentiating them is trivial. Differentiating the commutators with $Q(\lambda)$ is done by means of (II.11). The arguments of Proposition III.5 of [13] show that, algebraically,

$$
\frac{d}{d \lambda} \operatorname{Ch}(Q(\lambda))=(b+B) G^{\lambda}
$$

where $b$ and $B$ are the coboundary operators of entire cyclic cohomology. We will be finished if we prove that each $G_{2 k-1}^{\lambda}$ is well-defined and obeys the growth condition of entire cyclic cohomology.

The key estimate on $Q(\lambda)$ is

$$
\left\|Q(\lambda) e^{-s H}\right\| \leq C s^{-1 / 2}
$$


which follows from the spectral theorem. We proceed as in the derivation of (II.6), by applying Hölder's inequality to the trace in (II.12). We obtain the estimate

$$
\begin{aligned}
& \left|\operatorname{Tr}\left(A_{0} e^{-s_{0} H} A_{1} e^{-s_{1} H} \ldots Q^{\prime}(\lambda) e^{-s_{l} H} \ldots A_{2 k-1} e^{-s_{n} H}\right)\right| \\
& \quad \leq\left\|Q^{\prime}(\lambda) e^{-s_{l} H / 2}\right\| \operatorname{Tr}\left(e^{-H}\right)^{1-s_{l}} \operatorname{Tr}\left(e^{-H / 2}\right)^{s_{l}} \prod_{j=0}^{2 k-1}\left\|A_{j}\right\| \\
& \quad \leq C s_{l}{ }^{-1 / 2} \prod_{j=0}^{2 k-1}\left\|A_{j}\right\| .
\end{aligned}
$$

Using this estimate, the integral over $\sigma_{2 k}^{1}$ in (II.12) is well-defined and gives a factor of $\frac{1}{(2 k-1) !}$. The sum in (II.12) involves $2 k$ such terms, so the resulting bound is

$$
\left|G_{2 k-1}^{\lambda}\left(a_{0}, \ldots, a_{2 k-1}\right)\right| \leq C \frac{2 k}{(2 k-1) !}\left\|a_{0}\right\| \prod_{l=1}^{2 k-1}\left\|\left[Q(\lambda), a_{l}\right]\right\|
$$

where $C$ is independent of $k$. This shows that the growth condition on $G^{\lambda}$ is satisfied and so $G^{\lambda}$ is an entire cochain.

II.D. In fact, formula (II.4) defines a one parameter family of cocycles indexed by $\beta$ (we will refer to $\beta$ as the temperature because of the obvious analogy with quantum statistical mechanics). It is shown in [14] and [16] that the entire cyclic cohomology class of $\mathrm{Ch}^{\beta}(Q)$ is independent of $\beta$. It is thus natural to study the limit $\beta \rightarrow \infty$. We have the following theorem ([11], Section 2.2).

Theorem II.2. The zero temperature limits,

$$
\mathrm{Ch}_{2 k}^{\infty}(Q)\left(a_{0}, a_{1}, \ldots, a_{2 k}\right):=\lim _{\beta \rightarrow \infty} \mathrm{Ch}_{2 k}^{\beta}(Q)\left(a_{0}, a_{1}, \ldots, a_{2 k}\right)
$$

exist and define continuous $(2 k+1)$-linear functionals over the algebra $\mathcal{A}^{Q}$. Moreover,

$$
\mathrm{Ch}_{2 k}^{\infty}(Q)\left(a_{0}, a_{1}, \ldots, a_{2 k}\right)=\frac{(-1)^{k}}{k !} \operatorname{Str}\left\{P_{0} a_{0} P_{0} \Omega\left(a_{1}, a_{2}\right) P_{0} \ldots P_{0} \Omega\left(a_{2 k-1}, a_{2 k}\right)\right\}
$$

where $P_{0}$ is the orthogonal projection onto $\operatorname{Ker}(H)$, and where $\Omega(a, b)=a b-a P_{0} b$.

In the examples studied in this paper, $\operatorname{Ker}(Q)$ is a one dimensional subspace of the even part $\mathcal{H}_{0}$ of $\mathcal{H}$. The above theorem then yields the following corollary. 
Corollary II.3. Let $\operatorname{Ker}(Q)$ be a one dimensional subspace of $\mathcal{H}_{0}$ and let $a_{0}, a_{1}, \ldots, a_{2 k} \in$ $\mathcal{A}^{Q}$. Then

$$
\mathrm{Ch}_{2 k}^{\infty}(Q)\left(a_{0}, a_{1}, \ldots, a_{2 k}\right)=\frac{(-1)^{k}}{k !}\left\langle a_{0}\right\rangle_{0} \prod_{m=0}^{k}\left\{\left\langle a_{2 m-1} a_{2 m}\right\rangle_{0}-\left\langle a_{2 m-1}\right\rangle_{0}\left\langle a_{2 m}\right\rangle_{0}\right\}
$$

where $\langle a\rangle_{0}:=\left(\phi_{0}, a \phi_{0}\right)$, and where $\phi_{0}$ is a normalized vector spanning $\operatorname{Ker}(Q)$.

Remark. The factors $\langle a b\rangle_{0}-\langle a\rangle_{0}\langle b\rangle_{0}$ appearing in (II.19) are the truncated vacuum expectation values of $a$ and $b$.

Proof. As a consequence of Theorem II.2,

$$
\begin{aligned}
\mathrm{Ch}_{2 k}^{\infty}(Q) & \left(a_{0}, a_{1}, \ldots, a_{2 k}\right)= \\
& \frac{(-1)^{k}}{k !}\left(\phi_{0}, a_{0} P_{0} \Omega\left(a_{1}, a_{2}\right) P_{0} \ldots P_{0} \Omega\left(a_{2 k-1}, a_{2 k}\right) \phi_{0}\right)= \\
& \frac{(-1)^{k}}{k !}\left\langle a_{0}\right\rangle_{0} \prod_{m=0}^{k}\left\{\left\langle a_{2 m-1} a_{2 m}\right\rangle_{0}-\left\langle a_{2 m-1}\right\rangle_{0}\left\langle a_{2 m}\right\rangle_{0}\right\}
\end{aligned}
$$

as claimed.

Note, however, that the limit $\mathrm{Ch}^{\infty}(Q):=\left\{\mathrm{Ch}_{2 k}^{\infty}(Q)\right\}_{k=0}^{\infty}$ of $\mathrm{Ch}^{\beta}(Q)$ does not define an entire cyclic cocycle. The power series $\sum_{k \geq 0} k !\left\|\mathrm{Ch}_{2 k}^{\infty}(Q)\right\| z^{2 k}$ has a finite, rather than infinite, convergence radius, and so Connes' growth condition is violated. As a consequence, the usual pairing [10], [14], $<\mathrm{Ch}^{\infty}(Q), e>$ of $\mathrm{Ch}^{\infty}(Q)$ with a $K_{0}(\mathcal{A})$ class $e$ is meaningless. It is, however, easy to see that if a hermitian projection $e \in \operatorname{Mat}(\mathcal{A})$ is such that the operator $I-2 P_{0} e P_{0}$ is invertible, then the series defining $<\mathrm{Ch}^{\infty}(Q), e>$ converges and, in fact

$$
<\mathrm{Ch}^{\infty}(Q), e>=\frac{1}{2} \operatorname{Str}_{\operatorname{Ker}(Q)}\left\{I-\frac{I-2 P_{0} e P_{0}}{\left[\left(I-2 P_{0} e P_{0}\right)^{2}\right]^{1 / 2}}\right\}
$$

Note that $<\mathrm{Ch}^{\infty}(Q), e>$ is an integer such that $\left|<\mathrm{Ch}^{\infty}(Q), e>\right| \leq \operatorname{dim} \operatorname{Ker}(Q)$. We are not aware of a topological significance of this integer. 


\section{Fredholm Modules Over the Quantum Type I Cartan Domains}

III.A. The Cartan domains of type I form an infinite sequence $D_{m, n}, m, n \geq 1$, of noncompact hermitian symmetric spaces. $D_{m, n}$ is an open subset of $\mathbb{C}^{m n}$ defined as follows:

$$
D_{m, n}:=\left\{z \in \operatorname{Mat}_{m, n}(\mathbb{C}): I_{m}-z z^{*}>0\right\} .
$$

The quantum deformation of $D_{m, n}$ is the Toeplitz algebra $\mathcal{T}_{r}\left(D_{m, n}\right)$, defined as follows [7]. For $r>m+n-1$, we consider the following measure on $D_{m, n}$ :

$$
d \mu_{r}(z)=\Lambda_{r} \operatorname{det}\left(I_{m}-z z^{*}\right)^{r-(m+n)} d^{2 m n} z .
$$

The normalization factor $\Lambda_{r}$ is chosen to normalize the total integral to one:

$$
\Lambda_{r}=\pi^{-m n} \prod_{k=1}^{n} \frac{\Gamma(r-n+k)}{\Gamma(r-m-n+k)} .
$$

We let $\mathcal{H}_{r}(D)$ denote the Hilbert space of holomorphic functions on $D_{m, n}$ which are square integrable with respect to $d \mu_{r}$. The Bergman kernel of $D_{m, n}$ associated with this measure is given by $K^{r}(z, w)=\operatorname{det}\left(I_{m}-z w^{*}\right)^{-r}$. The algebra $\mathcal{T}_{r}\left(D_{m, n}\right)$ is the $\mathbb{C}^{*}$-algebra generated by the Toeplitz operators on $\mathcal{H}_{r}(D)$ whose symbols are smooth functions on $D_{m, n}$ which extend to the closure $\bar{D}_{m, n}$. Its generators $\sigma_{i j}:=T_{r}\left(z_{i j}\right)$ and $\bar{\sigma}_{i j}=T_{r}\left(\bar{z}_{i j}\right)$ obey the relations

$$
\begin{aligned}
& {\left[\bar{\sigma}_{i j}, \sigma_{k l}\right]=\mu\left(I-\sigma \sigma^{*}\right)_{k i}\left(I-\sigma^{*} \sigma\right)_{j l},} \\
& {\left[\sigma_{i j}, \sigma_{k l}\right]=0}
\end{aligned}
$$

where $\mu=1 /(r-m)$.

III.B. Our construction of Fredholm modules over $\mathcal{T}_{r}\left(D_{m, n}\right)$ will be based on a quantization of the type I Cartan superdomain $\mathcal{M} \equiv D_{m, n \mid n}^{I}[6]$. The starting point of this construction is the Hilbert space $\mathcal{H}_{r}(\mathcal{M})$ of superholomorphic functions on $\mathcal{M}$ which are square integrable with respect to the measure

$$
d \mu_{r}(Z)=\frac{1}{\pi^{m n}} \operatorname{det}\left(I_{m}-Z Z^{*}\right)^{r-m} d^{2 m n} z d^{2 m n} \theta .
$$

Here, $\theta$ denotes the matrix of fermionic generators and $Z=(z, \theta)$ is a collective matrix notation for the generators of $C^{\infty}(\mathcal{M})$. The corresponding Bergman kernel is given by

$$
K^{r}(Z, W)=\operatorname{det}\left(I_{m}-Z W^{*}\right)^{-r}
$$

Let $\mathcal{T}_{r}(\mathcal{M})$ denote the $\mathbb{C}^{*}$-algebra generated by the super Toeplitz operators on $\mathcal{H}_{r}(\mathcal{M})$ with smooth symbols extending to the boundary. Its generators are $\Sigma_{i j}=T_{r}\left(Z_{i j}\right)$ and $\bar{\Sigma}_{i j}=T_{r}\left(\bar{Z}_{i j}\right)$. Note that $\bar{\Sigma}_{i j}$ is the adjoint of $\Sigma_{i j}$. We will adopt a matrix notation: $\Sigma^{*}{ }_{i j}=\bar{\Sigma}_{j i}$. Often we will write $\Sigma=(\sigma, \chi)$ to indicate the submatrices of even and odd operators.

The theorem below applies to all Type I Cartan superdomains $\mathcal{D}$, not just the supersymmetric case that we have denoted by $\mathcal{M}$. 
Theorem III.1. Using the above notation, the generators of $\mathcal{T}_{r}(\mathcal{D})$, where $\mathcal{D}=D_{m, n \mid q}^{I}$ is an arbitrary type I Cartan superdomain, satisfy the following relations:

$$
\begin{aligned}
& {\left[\Sigma_{i j}, \Sigma_{k l}\right]=0} \\
& {\left[\bar{\Sigma}_{i j}, \Sigma_{k l}\right]=\mu\left(I_{m}-\Sigma \Sigma^{*}\right)_{k i}\left(I_{n \mid q}-\Sigma^{*} \Sigma\right)_{j l}}
\end{aligned}
$$

where $[\cdot, \cdot]$ is the graded commutator, and $\mu=1 /(r-m)$. In other words,

$$
\begin{aligned}
& {\left[\bar{\sigma}_{i j}, \sigma_{k l}\right]=\mu\left(I_{m}-\sigma \sigma^{*}-\chi \chi^{*}\right)_{k i}\left(I_{n}-\sigma^{*} \sigma\right)_{j l},} \\
& {\left[\bar{\sigma}_{i j}, \chi_{k l}\right]=-\mu\left(I_{m}-\sigma \sigma^{*}-\chi \chi^{*}\right)_{k i}\left(\sigma^{*} \chi\right)_{j l},} \\
& {\left[\bar{\chi}_{i j}, \chi_{k l}\right]=\mu\left(I_{m}-\sigma \sigma^{*}-\chi \chi^{*}\right)_{k i}\left(I_{n}-\chi^{*} \chi\right)_{j l} .}
\end{aligned}
$$

Before proving Theorem III.1, we first prove two lemmas. Let $\Delta_{i j}$ be the unbounded operator on $\mathcal{H}_{r}(\mathcal{D})$ given by

$$
\Delta_{i j} \phi(Z)=\frac{\partial}{\partial Z_{i j}} \phi(Z)
$$

Lemma III.2. For $\phi \in \mathcal{H}_{r}(\mathcal{D})$ in the domain of $\Delta_{i j}$ for all $1 \leq i \leq m$, we have

$$
\sum_{j=1}^{m} \Delta_{j k}\left(I_{m}-\Sigma \Sigma^{*}\right)_{j i} \phi=\mu^{-1} \bar{\Sigma}_{i k} \phi
$$

The domain of this operator thus extends to all of $\mathcal{H}_{r}(\mathcal{D})$.

Proof. By definition we have

$$
\sum_{j=1}^{m} \Delta_{j k}\left(I_{m}-\Sigma \Sigma^{*}\right)_{j i} \phi(Z)=\sum_{j=1}^{m} \int_{\mathcal{M}} \frac{\partial}{\partial Z_{j k}}\left[K^{r}(Z, Y)\left(I_{m}-Z Y^{*}\right)_{j i}\right] \phi(Y) d \mu_{r}(Y) .
$$

To evaluate the derivative, we need the fact that

$$
\frac{\partial}{\partial Z_{j k}} K^{r}(Z, Y)=r K^{r}(Z, Y)\left[Y^{*}\left(I_{m}-Z Y^{*}\right)^{-1}\right]_{k j} .
$$

We thus obtain

$$
\begin{aligned}
& \sum_{j=1}^{m} \frac{\partial}{\partial Z_{j k}}\left[K^{r}(Z, Y)\left(I_{m}-Z Y^{*}\right)_{j i}\right] \\
& \quad=K^{r}(Z, Y) \sum_{j=1}^{m}\left[r\left[Y^{*}\left(I_{m}-Z Y^{*}\right)^{-1}\right]_{k j}\left(I_{m}-Z Y^{*}\right)_{j i}-\bar{Y}_{i k}\right] \\
& \quad=(r-m) \bar{Y}_{i k} K^{r}(Z, Y) .
\end{aligned}
$$

The lemma follows. 
Lemma III.3. For $\phi \in \mathcal{H}_{r}(\mathcal{D})$ in the domain of $\Delta_{i j}$ for all $1 \leq j \leq n+q$, we have

$$
\sum_{k=1}^{n+q}\left(I_{n \mid q}-\Sigma^{*} \Sigma\right)_{j k} \Delta_{l k} \phi=\mu^{-1} \bar{\Sigma}_{l j} \phi
$$

The domain of this operator thus extends to all of $\mathcal{H}_{r}(\mathcal{D})$.

Proof. We start with

$$
\sum_{k=1}^{n+q}\left(I_{n \mid q}-\Sigma^{*} \Sigma\right)_{j k} \Delta_{l k} \phi(Z)=\sum_{k=1}^{n+q} \int_{\mathcal{M}} K^{r}(Z, W)\left(I_{n \mid q}-W^{*} W\right)_{j k} \frac{\partial}{\partial W_{l k}} \phi(W) d \mu_{r}(W) .
$$

Integrating by parts gives

$$
\begin{aligned}
& \sum_{k=1}^{n+q}\left(I_{n \mid q}-\Sigma^{*} \Sigma\right)_{j k} \Delta_{l k} \phi(Z) \\
& =-\sum_{k=1}^{n+q}(-1)^{\epsilon_{k}\left(\epsilon_{j}+1\right)} \int_{\mathcal{M}} K^{r}(Z, W) \frac{\partial}{\partial W_{l k}}\left[\left(I_{n \mid q}-W^{*} W\right)_{j k} \operatorname{det}\left(I_{n \mid q}-W^{*} W\right)^{r-m}\right] \phi(W) d W
\end{aligned}
$$

where $\epsilon_{j}:=p\left(Z_{i j}\right)$. The derivative is easily computed,

$$
\begin{aligned}
& \frac{\partial}{\partial W_{l k}}\left(I_{n \mid q}-W^{*} W\right)_{j k} \operatorname{det}\left(I_{n \mid q}-W^{*} W\right)^{r-m} \\
& =-\operatorname{det}\left(I_{n \mid q}-W^{*} W\right)^{r-m}\left[(-1)^{\epsilon_{k} \epsilon_{j}} \bar{W}_{l j}\right. \\
& \left.\quad+(-1)^{\epsilon_{k}\left(\epsilon_{j}-1\right)}(r-m)\left(I_{n \mid q}-W^{*} W\right)_{j k}\left[\left(I_{n \mid q}-W^{*} W\right)^{-1} W^{*}\right]_{k l}\right] .
\end{aligned}
$$

Summing over $k$ we obtain

$$
\begin{gathered}
-\sum_{k=1}^{n+q}(-1)^{\epsilon_{k}\left(\epsilon_{j}+1\right)} \frac{\partial}{\partial W_{l k}}\left(I_{n \mid q}-W^{*} W\right)_{j k} \operatorname{det}\left(I_{n \mid q}-W^{*} W\right)^{r-m} \\
=(r-m) \bar{W}_{l j} \operatorname{det}\left(I_{n \mid q}-W^{*} W\right)^{r-m} .
\end{gathered}
$$

In view of (III.16), this completes the proof.

Proof of Theorem III.1. We start with the fact that

$$
\left[\Delta_{a b}, \Sigma_{k l}\right]=\delta_{a k} \delta_{b l}
$$


restricted to the domain of $\delta_{i j}$. We apply operators to both sides of this equation and contract indices:

$$
\sum_{a, b}\left(I_{n \mid q}-\Sigma^{*} \Sigma\right)_{j b}\left[\Delta_{a b}, \Sigma_{k l}\right]\left(I_{m}-\Sigma \Sigma^{*}\right)_{a i}=\left(I_{n \mid q}-\Sigma^{*} \Sigma\right)_{l j}\left(I_{m}-\Sigma \Sigma^{*}\right)_{k i}
$$

Using Lemma III.2 and Lemma III.3, we reduce the right-hand side to

$$
\mu^{-1} \sum_{a} \bar{\Sigma}_{a j} \Sigma_{k l}\left(I_{m}-\Sigma \Sigma^{*}\right)_{a i}-\mu^{-1} \sum_{b}(-1)^{\epsilon_{b} \epsilon_{l}}\left(I_{n \mid q}-\Sigma^{*} \Sigma\right)_{j b} \Sigma_{k l} \bar{\Sigma}_{i b} \mu^{-1}\left[\bar{\Sigma}_{i j}, \Sigma_{k l}\right]
$$

This proves the theorem on a restricted domain. It is easy to see that this domain is dense, and since both sides of the relation are bounded operators, there is no problem in removing the restriction.

III.C. For $f \in C^{\infty}\left(D_{m, n}\right)$ bounded, the Toeplitz operator $T_{r}(f)$ defines a unique super Toeplitz operator which we will denote also by $T_{r}(f)$. This defines an action of $T_{r}(f)$ on the $\mathbb{Z}_{2}$-graded Hilbert space $\mathcal{H}_{r}(\mathcal{M})$. A continuity argument shows that this action extends to an action of the $\mathbb{C}^{*}$-algebra $\mathcal{T}_{r}\left(D_{m, n}\right)$ on $\mathcal{H}_{r}(\mathcal{M})$, and so we have a $*$-morphism $\rho: \mathcal{T}_{r}\left(D_{m, n}\right) \longrightarrow \mathcal{L}\left(\mathcal{H}_{r}(\mathcal{M})\right)$.

Let $\mathcal{P} \subset \mathcal{H}_{r}(D)$ denote the dense subspace spanned by all polynomials. We now take $\operatorname{Dom}\left(\Delta_{i j}\right)=\mathcal{P}$. This operator is broken up into its even and odd components as $\Delta=(\partial, \tau)$, where, if $Z=(z, \theta)$,

$$
\partial_{i j} \phi(Z)=\frac{\partial}{\partial z_{i j}} \phi(Z), \quad \tau_{i j} \phi(Z)=\frac{\partial}{\partial \theta_{i j}} \phi(Z) .
$$

Let $\bar{\Delta}_{i j}, \bar{\partial}_{i j}$, and $\bar{\tau}_{i j}$ denote the hermitian adjoints of $\Delta_{i j}, \partial_{i j}$, and $\tau_{i j}$, respectively. Now we define the operator

$$
d:=\sum_{i j} \chi_{i j} \partial_{i j}
$$

and let $d^{*}:=\sum_{i j} \bar{\chi}_{i j} \bar{\partial}_{i j}$ denote its adjoint.

The two operators

$$
\begin{aligned}
& Q_{1}:=d+d^{*}, \\
& Q_{2}:=i\left(d-d^{*}\right),
\end{aligned}
$$

are defined on $\mathcal{P}$ and symmetric. Let $N_{0}$ and $N_{1}$ denote the operators on $\mathcal{P}$,

$$
\begin{aligned}
& N_{0}:=\sum_{i, j} \sigma_{i j} \partial_{i j}, \\
& N_{1}:=\sum_{i, j} \chi_{i j} \tau_{i j} .
\end{aligned}
$$


Note that on monomials these operators have the form

$$
\begin{aligned}
& N_{0}\left(z^{\mu} \theta^{\alpha}\right):=|\mu| z^{\mu} \theta^{\alpha}, \\
& N_{1}\left(z^{\mu} \theta^{\alpha}\right):=|\alpha| z^{\mu} \theta^{\alpha},
\end{aligned}
$$

where $\mu \in \mathbb{Z}_{+}^{m n}$ and $\alpha \in\{0,1\}^{m n}$ are multi-indices and $|\mu|=\mu_{1}+\ldots+\mu_{m n}$. Let $H$ be the total number operator,

$$
H:=N_{0}+N_{1}
$$

Then $N_{0}$ is symmetric and $N_{1}$ is bounded and self-adjoint.

Proposition III.4. As operators on $\mathcal{P}$,

$$
Q_{1}^{2}=Q_{2}^{2}=H
$$

Proof. Using Lemma III.2 we have

$$
\begin{aligned}
d^{*} & =\mu \sum_{i, j, k} \tau_{k j}\left(I_{m}-\Sigma \Sigma^{*}\right)_{k i} \bar{\partial}_{i j} \\
& =\sum_{j, k} \sigma_{j k} \tau_{j k} .
\end{aligned}
$$

Thus,

$$
Q_{1}=\sum_{j, k} \chi_{j k} \partial_{j k}+\sigma_{j k} \tau_{j k}
$$

and

$$
\begin{aligned}
Q_{1}^{2} & =\sum_{i, j, k, l}\left[\chi_{i j} \partial_{i j}, \sigma_{k l} \tau_{k l}\right] \\
& =\sum_{i, j, k, l} \chi_{i j}\left[\partial_{i j}, \sigma_{k l}\right] \tau_{k l}+\sigma_{k l}\left[\chi_{i j}, \tau_{k l}\right] \partial_{i j} \\
& =N_{1}+N_{0} .
\end{aligned}
$$

The proof for $Q_{2}$ is essentially identical.

\section{Proposition III.5.}

(i) The operators $Q_{j}, H$, and $N_{0}$ are essentially self-adjoint on $\mathcal{P}$.

(ii) For any $\epsilon>0,(H+I)^{-1 / 2} \in I_{2 m n+\epsilon}\left(\mathcal{H}_{r}(\mathcal{M})\right)$.

Proof. (i) Let $\phi \in \mathcal{P}$ be a polynomial of degree $m$. Then $\left\|H^{k} \phi\right\| \leq C m^{k}$, with $C$ independent of $k$. As a consequence, each $\phi \in \mathcal{P}$ is an analytic vector for $H$, and thus $H$ is essentially self-adjoint on $\mathcal{P}$ by Nelson's theorem [18]. Since $N_{0}=H-N_{1}$, with 
$N_{1}$ bounded, the same is true for $N_{0}$. Finally, $Q_{j}$ is essentially self-adjoint on $\mathcal{P}$ as $\left\|Q_{j}^{k} \phi\right\| \leq C m^{k / 2}$ for all $\phi \in \mathcal{P}$.

(ii) The spectrum of $N_{0}$ consists of the eigenvalues $\lambda_{p}=p, p=0,1,2, \ldots$ each of which has multiplicity not exceeding $2^{m n} \times$ the number of monomials in $z_{i j}$ of degree $p$ $=O\left(p^{m n-1}\right)$. Since $N_{1}$ is bounded, the claim follows.

The following proposition states that the operators $Q_{1}$ and $Q_{2}$ generate an $N=2$ supersymmetry algebra.

Proposition III.6. As operators on $\mathcal{P}$, we have the following relations:

$$
\begin{aligned}
& {\left[Q_{1}, \sigma_{i j}\right]=\chi_{i j}, \quad\left[Q_{1}, \bar{\sigma}_{i j}\right]=-\bar{\chi}_{i j}, \quad\left[Q_{1}, \chi_{i j}\right]=-\sigma_{i j}, \quad\left[Q_{1}, \bar{\chi}_{i j}\right]=\bar{\sigma}_{i j}} \\
& {\left[Q_{2}, \sigma_{i j}\right]=i \chi_{i j}, \quad\left[Q_{2}, \bar{\sigma}_{i j}\right]=i \bar{\chi}_{i j}, \quad\left[Q_{2}, \chi_{i j}\right]=i \sigma_{i j}, \quad\left[Q_{2}, \bar{\chi}_{i j}\right]=i \bar{\sigma}_{i j} .}
\end{aligned}
$$

Furthermore, the operators $Q_{j}$ satisfy the relations:

$$
\left[Q_{j}, Q_{k}\right]=2 \delta_{j k} H .
$$

Proof. We have trivially the following relations:

$$
\begin{aligned}
& {\left[d, \sigma_{i j}\right]=\chi_{i j},} \\
& {\left[d, \chi_{i j}\right]=0,} \\
& {\left[d, \bar{\chi}_{i j}\right]=\bar{\sigma}_{i j},}
\end{aligned}
$$

In addition, we see using the adjoint of (III.29) that

$$
\left[d, \bar{\sigma}_{i j}\right]=0,
$$

and

$$
\left[d, \bar{\chi}_{i j}\right]=\bar{\sigma}_{i j} .
$$

The relations (III.32) follow from (III.34), (III.35), and (III.36).

Among the relations (III.33), only $\left[Q_{1}, Q_{2}\right]=0$ needs to be established. This, however, is an immediate consequence of (III.24).

As a consequence of the above considerations and of Theorem II.1, we obtain the following theorem.

\section{Theorem III.7.}

(i) The two triples $\left(\mathcal{H}_{r}(\mathcal{M}), \rho, Q_{j}\right)$ define $2 m n$-summable Fredholm modules over $\mathcal{T}_{r}\left(D_{m, n}\right)$.

(ii) The corresponding Chern characters define the same cohomology class in the entire cyclic cohomology of $\mathcal{T}_{r}\left(D_{m, n}\right)^{1}$. 


\section{The Chern Characters}

IV.A. Let $\mathcal{A}^{\text {pol }}$ be the subalgebra of $\mathcal{T}_{r}\left(D_{m, n}\right)$ consisting of polynomials in the Toeplitz operators. Clearly, an element of $a \in \mathcal{A}^{\text {pol }}$ has a representation by an integral kernel:

$$
a \phi(Z)=\int_{\mathcal{M}} a(Z, W) \phi(W)
$$

where $a(Z, W)$ is an even function (with respect to the grading) which is holomorphic in $Z$ and depends smoothly on $W$. In this section we derive explicit expressions for $\mathrm{Ch}_{2 k}^{\beta}\left(Q_{j}\right)\left(a_{0}, \ldots, a_{2 k}\right)$, for $a_{j} \in \mathcal{A}^{\text {pol }}$, and give representations for these functionals in terms of multiple Berezin integrals over $\mathcal{M}$. We also consider the special case when the $a_{j}$ are Toeplitz operators.

For the remainder of this subsection, $f$ denotes a smooth function on $D_{m, n}$ whose first derivatives are bounded.

Proposition IV.1. For $f$ as above,

$$
\begin{aligned}
& \sum_{i=1}^{m}\left[\chi_{i j} \partial_{i k}, T_{r}(f)\right]=\sum_{i=1}^{m} T_{r}\left(\frac{\partial f}{\partial z_{i k}}\right) \chi_{i j} \\
& \sum_{i=1}^{m}\left[\bar{\chi}_{i j} \bar{\partial}_{i k}, T_{r}(f)\right]=-\sum_{i=1}^{m} \bar{\chi}_{i j} T_{r}\left(\frac{\partial f}{\partial \bar{z}_{i k}}\right) .
\end{aligned}
$$

Proof. It is sufficient to prove the first of these identities as the second one follows by taking the hermitian conjugate. Consider the commutator:

$$
\begin{aligned}
\sum_{i=1}^{m}\left[\chi_{i j} \partial_{i k}, T_{r}(f)\right]= & \sum_{i=1}^{m} \int_{\mathcal{M}} \theta_{i j} \frac{\partial}{\partial z_{i k}} K^{r}(Z, W) f(w) \phi(W) d \mu_{r}(W) \\
& +\sum_{i=1}^{m} \int_{\mathcal{M}} K^{r}(Z, W) \frac{\partial f}{\partial w_{i k}}(w) \eta_{i j} \phi(W) d \mu_{r}(W) \\
& +\sum_{i=1}^{m} \int_{\mathcal{M}} K^{r}(Z, W) f(w) \eta_{i j} \phi(W) \frac{\partial}{\partial w_{i k}} d \mu_{r}(W) .
\end{aligned}
$$

We use

$$
\frac{\partial}{\partial w_{i k}} \log \operatorname{det}\left(I_{m}-W W^{*}\right)^{-m}=m \sum_{l=1}^{m}\left(I_{m}-W W^{*}\right)^{-1}{ }_{l i} \bar{w}_{l k}
$$

to rewrite the third term of (IV.3) as

$$
m \sum_{i, l} \int_{\mathcal{M}} K^{r}(Z, W) f(w) \eta_{i j} \bar{w}_{l k}\left(I_{m}-W W^{*}\right)^{-1}{ }_{l i} \phi(W) d \mu_{r}(W) .
$$


We make the $\eta_{i j}$ into a derivative using

$$
\frac{\partial}{\partial \bar{\eta}_{l j}} \log \operatorname{det}\left(I_{m}-W W^{*}\right)^{-m}=-m \sum_{i=1}^{m}\left(I_{m}-W W^{*}\right)^{-1}{ }_{l i} \eta_{i j}
$$

The third term of (IV.3) thus becomes

$$
-(-1)^{p(\phi)} \sum_{l=1}^{m} \int_{\mathcal{M}} K^{r}(Z, W) f(w) \bar{w}_{l k} \phi(W) \frac{\partial}{\partial \bar{\eta}_{l j}} d \mu_{r}(W),
$$

where $p(\phi)$ is the parity of $\phi$, which appears because the $\eta$ was moved past the $\phi$. If we integrate by parts, this parity is cancelled and the third term of (IV.3) becomes

$$
\sum_{l=1}^{m} \int_{\mathcal{M}} \frac{\partial}{\partial \bar{\eta}_{l j}} K^{r}(Z, W) f(w) \bar{w}_{l k} \phi(W) d \mu_{r}(W)
$$

where the derivative strikes only the kernel. Using computations essentially identical to (IV.4) and (IV.6), we find that

$$
\sum_{l=1}^{m} \bar{w}_{l k} \frac{\partial}{\partial \eta_{l j}} K^{r}(Z, W)=-\sum_{i=1}^{m} \theta_{i j} \frac{\partial}{\partial w_{i k}} K^{r}(Z, W)
$$

Thus, the third term in (IV.3) cancels the first term, and the proposition follows.

Corollary IV.2. With the above definitions,

$$
\begin{gathered}
{\left[Q_{1}, T_{r}(f)\right]=\sum_{i, j} T_{r}\left(\frac{\partial f}{\partial z_{i j}}\right) \chi_{i j}-\bar{\chi}_{i j} T_{r}\left(\frac{\partial f}{\partial \bar{z}_{i j}}\right),} \\
{\left[Q_{2}, T_{r}(f)\right]=\sum_{i, j} i T_{r}\left(\frac{\partial f}{\partial z_{i j}}\right) \chi_{i j}+i \bar{\chi}_{i j} T_{r}\left(\frac{\partial f}{\partial \bar{z}_{i j}}\right) .}
\end{gathered}
$$

Corollary IV.3. There is an inclusion $\mathcal{A}^{\text {pol }} \subset \mathcal{T}_{r}\left(D_{m, n}\right)^{1}$.

IV.B. For $t \in \sigma_{n}^{\beta}$ and $a_{0}, \ldots, a_{n} \in \mathcal{A}^{\text {pol }}$ bounded, we now consider the expression

$$
\operatorname{Str}\left\{a_{0} a_{1}\left(t_{1}\right) \ldots a_{n}\left(t_{n}\right) e^{-\beta H}\right\}
$$

As a consequence of (II.6), the supertrace (IV.11) is well defined. Our goal in this subsection is to express it as a multiple integral over $\mathcal{M}$. The integral representation given below has the flavor of a Feynman-Kac representation in Euclidean field theory. 
Proposition IV.4. Under the above assumptions,

$$
\begin{aligned}
\operatorname{Str}\{ & \left.a_{0} a_{1}\left(t_{1}\right) \ldots a_{n}\left(t_{n}\right) e^{-\beta H}\right\} \\
= & \int_{\mathcal{M}^{n+1}} a_{0}\left(e^{-\left(\beta-t_{n}\right)} Z_{n}, Z_{0}\right) a_{1}\left(e^{-t_{1}} Z_{0}, Z_{1}\right) a_{2}\left(e^{-\left(t_{2}-t_{1}\right)} Z_{1}, Z_{2}\right) \ldots \\
& \ldots a_{n}\left(e^{-\left(t_{n}-t_{n-1}\right)} Z_{n-1}, Z_{n}\right) d \mu_{r}\left(Z_{0}\right) \ldots d \mu_{r}\left(Z_{n}\right) .
\end{aligned}
$$

Proof. Using a basis of homogeneous polynomials, we can write (IV.11) as

$$
\sum_{\alpha}(-1)^{p\left(\phi_{\alpha}\right)}\left(\phi_{\alpha}, a_{0} a_{1}\left(t_{1}\right) \ldots a_{n}\left(t_{n}\right) \phi_{\alpha}\right) e^{-\beta\left(\operatorname{deg} \phi_{\alpha}\right)} .
$$

Now, for $t>0$ and a holomorphic function $\phi \in \operatorname{Ran}\left(e^{-t H}\right)$, we clearly have

$$
e^{t H} \phi(Z)=\phi\left(e^{t} Z\right)
$$

by the definition of $H$. This, in turn, implies that

$$
a(t) \phi(Z)=\int_{\mathcal{M}} a\left(e^{-t} Z, W\right) \phi\left(e^{t} W\right) d \mu_{r}(W) .
$$

Using this fact we can rewrite (IV.13) as

$$
\begin{aligned}
& \sum_{\alpha}(-1)^{p\left(\phi_{\alpha}\right)} \int_{\mathcal{M}^{n+2}} \overline{\phi_{\alpha}\left(e^{-\beta} W\right)} a_{0}\left(W, Z_{0}\right) a_{1}\left(e^{-t_{1}} Z_{0}, Z_{1}\right) a_{2}\left(e^{-\left(t_{2}-t_{1}\right)} Z_{1}, Z_{2}\right) \ldots \\
& \quad \ldots a_{n}\left(e^{-\left(t_{n}-t_{n-1}\right)} Z_{n-1}, Z_{n}\right) \phi_{\alpha}\left(e^{t_{n}} Z_{n}\right) d \mu_{r}(W) d \mu_{r}\left(Z_{0}\right) \ldots d \mu_{r}\left(Z_{n}\right) \\
& =\sum_{\alpha} \int_{\mathcal{M}^{n+2}} a_{0}\left(W, Z_{0}\right) a_{1}\left(e^{-t_{1}} Z_{0}, Z_{1}\right) a_{2}\left(e^{-\left(t_{2}-t_{1}\right)} Z_{1}, Z_{2}\right) \ldots a_{l}\left(e^{-\left(t_{n}-t_{n-1}\right)} Z_{n-1}, Z_{n}\right) \\
& \quad \times \phi_{\alpha}\left(e^{t_{n}} Z_{n}\right) \overline{\phi_{\alpha}\left(e^{-\beta} W\right)} d \mu_{r}(W) d \mu_{r}\left(Z_{0}\right) \ldots d \mu_{r}\left(Z_{n}\right) .
\end{aligned}
$$

Because

$$
K^{r}(Z, W)=\sum_{\alpha} \phi_{\alpha}(Z) \overline{\phi_{\alpha}(W)}
$$

(IV.16) reduces to

$$
\begin{aligned}
& \int_{\mathcal{M}^{n+2}} a_{0}\left(W, Z_{0}\right) a_{1}\left(e^{-t_{1}} Z_{0}, Z_{1}\right) a_{2}\left(e^{-\left(t_{2}-t_{1}\right)} Z_{1}, Z_{2}\right) \ldots a_{n}\left(e^{-\left(t_{n}-t_{n-1}\right)} Z_{n-1}, Z_{n}\right) \\
& \times K^{r}\left(e^{-\left(\beta-t_{n}\right)} Z_{n}, W\right) d \mu_{r}(W) d \mu_{r}\left(Z_{0}\right) \ldots d \mu_{r}\left(Z_{n}\right) .
\end{aligned}
$$

We perform the $W$ integration, yielding (IV.12).

We now give the explicit formula for the Chern character. Here as in Section II, $\mathrm{Ch}_{2 k}^{\beta}\left(Q_{j}\right)$ denotes the $2 k$-th component of the Chern character associated with the Dirac operator $Q_{j}$. 
Theorem IV.5. Let $f_{0}, \ldots, f_{2 k} \in C^{\infty}\left(D_{m, n}\right)$ have bounded first derivatives. On $C^{\infty}(\mathcal{M})$ define the differential operator

$$
\theta \cdot \frac{\partial}{\partial z}:=\sum_{i j} \theta_{i j} \frac{\partial}{\partial z_{i j}}
$$

Then, for $j=1,2$ we have the integral representation:

$$
\begin{aligned}
\mathrm{Ch}_{2 k}^{\beta}\left(Q_{j}\right)\left(T_{r}\left(f_{0}\right), \ldots, T_{r}\left(f_{2 k}\right)\right) \\
=(-\beta)^{-k} \int_{I_{2 k+1}^{\beta}} \int_{\mathcal{M}^{2 k+1}} f_{0}\left(z_{0}\right) \prod_{m=1}^{2 k}\left\{\theta_{m} \cdot \frac{\partial}{\partial z_{m}} f_{m}\left(z_{m}\right)+(-1)^{j} \bar{\theta}_{m} \cdot \frac{\partial}{\partial \bar{z}_{m}} f_{m}\left(z_{m}\right)\right\} \\
\quad \times \prod_{l=0}^{2 k} K^{r}\left(e^{-s_{l}} Z_{l}, Z_{l+1}\right) d \mu_{r}\left(Z_{l}\right) \delta\left(\beta-\sum_{l=0}^{2 k} s_{l}\right) d^{2 k+1} s,
\end{aligned}
$$

where $Z_{2 k+1}:=Z_{0}$.

Proof. Start with the definition (II.4). The variables $t_{j}$ are replaced by $s_{j}:=t_{j+1}-t_{j}$. We use then the integral representation following directly from Proposition IV.4. We apply then Corollary IV.2 to conclude the proof.

\section{Fredholm Modules Over Quantized Vector Spaces}

V.A. A complex vector space $V \cong \mathbb{C}^{n}$ has a quantum deformation given by a Toeplitz $\mathbb{C}^{*}$-algebra (see [8] and references therein). The perturbed measure on $V$ is defined by $d \mu_{r}(z):=\frac{r^{n}}{\pi^{n}} \exp (-r z \cdot \bar{z}) d^{2 n} z$ on $V$, for $r>0$, where $z \cdot \bar{z}=\sum_{j} z_{j} \bar{z}_{j}$. Let $\mathcal{H}_{r}(V)$ be the Hilbert space of holomorphic functions on $V$ which are square integrable with respect to $d \mu_{r}$. The Bergman kernel for $d \mu_{r}$ on $V$ is

$$
K_{V}^{r}(z, w)=\exp (r z \cdot \bar{w})
$$

The Toeplitz algebra $\mathcal{T}_{r}(V)$ is the $\mathbb{C}^{*}$-algebra generated by the Toeplitz operators on $\mathcal{H}_{r}(V)$ whose symbols are smooth bounded functions on $V$. Its "generators" $\sigma_{i}=T_{r}\left(z_{i}\right)$ and $\bar{\sigma}_{i}=T_{r}\left(\bar{z}_{i}\right), 1 \leq i \leq n$ obey the relations

$$
\left[\bar{\sigma}_{j}, \sigma_{k}\right]=\frac{1}{r} \delta_{j k} .
$$

Note that $\sigma$ and $\bar{\sigma}$ are not bounded, so the algebra $\mathcal{T}_{r}(V)$ will be generated only by certain bounded functions of these operators. This issue will not be important here. 
To construct Fredholm modules over $\mathcal{T}_{r}(V)$ we proceed as in Section III.The supervector space $\mathcal{X} \cong \mathbb{C}^{n \mid n}$ is the supersymmetric version of $V$. The quantum deformation of $\mathcal{X}[5]$ is based on the Hilbert space $\mathcal{H}_{r}(\mathcal{X})$ of superholomorphic functions on $\mathcal{X}$ which are square integrable with respect to the measure $d \mu_{r}(Z):=\frac{1}{\pi} \exp (-r Z \cdot \bar{Z}) d^{2 n} z d^{2 n} \theta$. Here $\theta_{i}, 1 \leq i \leq n$ denote the fermionic generators, $Z=(z, \theta)$, and $Z \cdot \bar{Z}=\sum_{j}\left(z_{j} \bar{z}_{j}+\theta_{j} \bar{\theta}_{j}\right)$. The Bergman kernel for the measure $d \mu_{r}$ on $\mathcal{X}$ is

$$
K^{r}(Z, W)=\exp (r Z \cdot \bar{W})
$$

We denote the algebra generated by super Toeplitz operators on $\mathcal{H}_{r}(\mathcal{X})$ with smooth bounded symbols on $\mathcal{X}$ by $\mathcal{T}_{r}(\mathcal{X})$. We define "generators" $\sigma_{i}:=T_{r}\left(z_{i}\right), \bar{\sigma}_{i}:=T_{r}\left(\bar{z}_{i}\right)$, $\chi_{i}:=T_{r}\left(\theta_{i}\right)$ and $\bar{\chi}_{i}:=T_{r}\left(\bar{\theta}_{i}\right)$. The operators $\sigma_{i}$ and $\bar{\sigma}_{i}$ are not elements of $\mathcal{T}_{r}(\mathcal{X})$, and they will be interpreted as unbounded operators on $\mathcal{H}_{r}(\mathcal{X})$. The generators satisfy the relations:

$$
\begin{aligned}
{\left[\bar{\sigma}_{j}, \sigma_{k}\right] } & =\frac{1}{r} \delta_{j k}, \\
{\left[\bar{\chi}_{j}, \sigma_{k}\right] } & =0 \\
{\left[\bar{\chi}_{j}, \chi_{k}\right] } & =\frac{1}{r} \delta_{j k}, \\
{\left[\sigma_{j}, \sigma_{k}\right] } & =\left[\chi_{j}, \chi_{k}\right]=\left[\sigma_{j}, \chi_{k}\right]=0,
\end{aligned}
$$


and their hermitian conjugates.

V.B. We can decompose the Hilbert space $\mathcal{H}_{r}(\mathcal{X})$ into odd and even subspaces, which are orthogonal. As in Section III, we can define a grading preserving $*$-morphism $\rho: \mathcal{T}_{r}(V) \rightarrow$ $\mathcal{L}\left(\mathcal{H}_{r}(\mathcal{X})\right)$. We again let $\mathcal{P} \subset \mathcal{H}_{r}(\mathcal{X})$ denote the dense subspace spanned by polynomials in $z$ and $\theta$. For $1 \leq i \leq n$ we define the operator

$$
\partial_{i} \phi(Z)=\frac{\partial}{\partial z_{i}} \phi(Z)
$$

with $\operatorname{Dom}\left(\partial_{i}\right)=\mathcal{P}$, and let $\bar{\partial}_{i}$ denote its hermitian adjoint. Let

$$
d:=\sum_{j} \chi_{j} \partial_{j}
$$

with adjoint $d^{*}$. Then the two operators

$$
\begin{aligned}
& Q_{1}:=d+d^{*}, \\
& Q_{2}:=i\left(d-d^{*}\right),
\end{aligned}
$$

are defined on $\mathcal{P}$ and symmetric. Let $N_{0}$ and $N_{1}$ denote the following operators on $\mathcal{P}$ :

$$
\begin{aligned}
& N_{0}\left(z^{\mu} \theta^{\alpha}\right):=|\mu| z^{\mu} \theta^{\alpha}, \\
& N_{1}\left(z^{\mu} \theta^{\alpha}\right):=|\alpha| z^{\mu} \theta^{\alpha},
\end{aligned}
$$

where $\mu$ and $\alpha$ are multi-indices. $N_{0}$ is symmetric, $N_{1}$ is bounded and self-adjoint, and we let $H:=N_{0}+N_{1}$.

Proposition V.1. As operators on $\mathcal{P}$,

$$
Q_{1}^{2}=Q_{2}^{2}=H
$$

In other words, $H$ is the Laplace operator corresponding to both $Q_{1}$ and $Q_{2}$.

Proof. We make use of the orthonormal basis for $\mathcal{H}_{r}(\mathcal{X})[5]$,

$$
\phi_{\mu, \alpha}(Z):=\left(\frac{r^{|\mu|+|\alpha|}}{\mu !}\right)^{1 / 2} z^{\mu} \theta^{\alpha},
$$

where $\mu !=\mu_{1} ! \ldots \mu_{n} !$ and $\theta^{\alpha}$ is ordered $\theta_{1}^{\alpha_{1}} \ldots \theta_{n}^{\alpha_{n}}$. We easily derive

$$
\begin{aligned}
\sigma_{j} \phi_{\mu, \alpha} & =\left[\left(\mu_{j}+1\right) / r\right]^{1 / 2} \phi_{\mu+1_{j}, \alpha}, \\
\bar{\sigma}_{j} \phi_{\mu, \alpha} & =\left(\mu_{j} / r\right)^{1 / 2} \phi_{\mu-1_{j}, \alpha}, \\
\chi_{j} \phi_{\mu, \alpha} & =(-1)^{\left\{\sum_{k<j} \alpha_{k}\right\}}\left(1-\alpha_{j}\right) r^{-1 / 2} \phi_{\mu, \alpha+1_{j}}, \\
\bar{\chi}_{j} \phi_{\mu, \alpha} & =(-1)^{\left\{\sum_{k<j} \alpha_{k}\right\}} \alpha_{j} r^{-1 / 2} \phi_{\mu, \alpha-1_{j}}, \\
\partial_{j} \phi_{\mu, \alpha} & =\left(\mu_{j} r\right)^{1 / 2} \phi_{\mu-1_{j}, \alpha}, \\
\bar{\partial}_{j} \phi_{\mu, \alpha} & =\left[\left(\mu_{j}+1\right) r\right]^{1 / 2} \phi_{\mu+1_{j}, \alpha},
\end{aligned}
$$


where $1_{j}$ is the multi-index with 1 in the $j$-th place and zeroes elsewhere. We compute using (V.11):

$$
\begin{aligned}
Q_{j}^{2} & =\left[d, d^{*}\right] \\
& =\sum_{k, l}\left\{\chi_{l} \bar{\chi}_{k}\left[\partial_{l}, \bar{\partial}_{k}\right]+\left[\chi_{l}, \bar{\chi}_{k}\right] \bar{\partial}_{k} \partial_{l}\right\} \\
& =\sum_{k}\left\{r \chi_{k} \bar{\chi}_{k}+\frac{1}{r} \bar{\partial}_{k} \partial_{k}\right\} \\
& =N_{1}+N_{0},
\end{aligned}
$$

for $j=1,2$.

\section{Proposition V.2.}

(i) The operators $Q_{j}, H$ and $N_{0}$ are essentially self-adjoint on $\mathcal{P}$.

(ii) For any $\epsilon>0,(H+I)^{-1 / 2} \in I_{2 n+\epsilon}\left(\mathcal{H}_{r}(\mathcal{X})\right)$.

Proof. The proof (i) follows that of Proposition III.5 (i). For (ii) we observe that the norm

$$
\begin{aligned}
\left\|(H+I)^{-1 / 2}\right\|_{2 l+\epsilon}^{2} & =\sum_{\mu, \alpha}(1+|\mu|+|\alpha|)^{-l-\epsilon / 2} \\
& \leq n \sum_{\mu}(1+|\mu|)^{-l-\epsilon / 2}
\end{aligned}
$$

is finite for all $\epsilon>0$ precisely when $l=n$.

The following proposition states that the operators $Q_{j}$ with $j=1,2$ generate an $N=2$ supersymmetry algebra.

Proposition V.3. As operators on $\mathcal{P}$, we have the following relations

$$
\begin{aligned}
& {\left[Q_{1}, \sigma_{j}\right]=\chi_{j}, \quad\left[Q_{1}, \bar{\sigma}_{j}\right]=-\bar{\chi}_{j}, \quad\left[Q_{1}, \chi_{j}\right]=-\sigma_{j}, \quad\left[Q_{1}, \bar{\chi}_{j}\right]=\bar{\sigma}_{j},} \\
& {\left[Q_{2}, \sigma_{j}\right]=i \chi_{j}, \quad\left[Q_{2}, \bar{\sigma}_{j}\right]=i \bar{\chi}_{j}, \quad\left[Q_{2}, \chi_{j}\right]=i \sigma_{j}, \quad\left[Q_{2}, \bar{\chi}_{j}\right]=i \bar{\sigma}_{j} .}
\end{aligned}
$$

Furthermore, the operators $Q_{j}$ satisfy

$$
\left[Q_{j}, Q_{k}\right]=2 \delta_{j k} H
$$

Proof. These are easily derived from (V.11).

Following the arguments of Theorem III.7 we establish the following result.

\section{Theorem V.4.}

(i) The two triples $\left(\mathcal{H}_{r}(\mathcal{X}), \rho, Q_{j}\right)$ define $2 n$-summable Fredholm modules over $\mathcal{T}_{r}(V)$.

(ii) The corresponding Chern characters define the same cohomology class in the entire cyclic cohomology of $\mathcal{T}_{r}(V)^{1}$. 
V.C. We now proceed to find explicit expressions for the Chern characters, following the approach of Section IV.

Proposition V.5. For $f \in C^{\infty}(V)$ bounded with bounded first derivative, we have the relations

$$
\begin{aligned}
& {\left[\partial_{j}, T_{r}(f)\right]=T_{r}\left(\frac{\partial}{\partial z_{j}} f\right),} \\
& {\left[\chi_{j}, T_{r}(f)\right]=0,}
\end{aligned}
$$

and their complex conjugates.

Proof. The second property follows immediately from the factorization

$$
d \mu_{r}(Z)=\exp (-r z \cdot \bar{z}) \exp (-r \theta \cdot \bar{\theta}) d^{2 n} z d^{2 n} \theta
$$

To prove the first we evaluate

$$
\begin{aligned}
\partial_{j} T_{r}(f) \phi(Z) & =\int_{\mathcal{X}} \frac{\partial}{\partial z_{j}} K^{r}(Z, W) f(w) \phi(W) d \mu_{r}(W) \\
& =r \int_{\mathcal{X}} \bar{w}_{j} K^{r}(Z, W) f(w) \phi(W) d \mu_{r}(W)
\end{aligned}
$$

We also have

$$
\begin{aligned}
T_{r}(f) \partial_{j} \phi(Z)= & \int_{\mathcal{X}} K^{r}(Z, W) f(w) \frac{\partial}{\partial w_{j}} \phi(W) d \mu_{r}(W) \\
= & r \int_{\mathcal{X}} K^{r}(Z, W) f(w) \phi(W) \bar{w}_{j} d \mu_{r}(W) \\
& \quad-\int_{\mathcal{X}} K^{r}(Z, W) \frac{\partial}{\partial w_{j}} f(w) \phi(W) d \mu_{r}(W),
\end{aligned}
$$

using an integration by parts. This completes the proof.

Corollary V.6. For $f \in C^{\infty}(V)$ bounded with bounded first derivatives, we have the relations

$$
\begin{aligned}
& {\left[Q_{1}, T_{r}(f)\right]=\sum_{j} T_{r}\left(\frac{\partial f}{\partial z_{j}} \theta_{j}-\frac{\partial f}{\partial z_{j}} \bar{\theta}_{j}\right)} \\
& {\left[Q_{2}, T_{r}(f)\right]=i \sum_{j} T_{r}\left(\frac{\partial f}{\partial z_{j}} \theta_{j}+\frac{\partial f}{\partial z_{j}} \bar{\theta}_{j}\right) .}
\end{aligned}
$$


V.D. For $t \in \sigma_{m}^{\beta}$ and $a_{0}, \ldots, a_{m} \in \mathcal{A}^{\text {pol }}$, we form the supertrace

$$
\operatorname{Str}\left\{a_{0} a_{1}\left(t_{1}\right) \ldots a_{m}\left(t_{m}\right) e^{-\beta H}\right\}
$$

which is well-defined because of (II.6).

Proposition V.7. Under the above assumptions,

$$
\begin{aligned}
\operatorname{Str} & \left\{a_{0} a_{1}\left(t_{1}\right) \ldots a_{n}\left(t_{n}\right) e^{-\beta H}\right\} \\
= & \int_{\mathcal{X}^{n+1}} a_{0}\left(e^{-t_{1}} Z_{0}, Z_{1}\right) a\left(e^{-\left(t_{2}-t_{1}\right)} Z_{1}, Z_{2}\right) \ldots a\left(e^{-\left(t_{n}-t_{n-1}\right)} Z_{n-1}, Z_{n}\right) \\
& \quad \times K^{r}\left(e^{-\left(\beta-t_{n}\right)} Z_{n}, Z_{0}\right) d \mu_{r}\left(Z_{0}\right) \ldots d \mu_{r}\left(Z_{n}\right) .
\end{aligned}
$$

Proof. Using the basis (V.10), we can write (V.21) as

$$
\sum_{\mu, \alpha}(-1)^{|\alpha|}\left(\phi_{\mu, \alpha}, a_{0} a_{1}\left(t_{1}\right) \ldots a_{m}\left(t_{m}\right) \phi_{\mu, \alpha}\right) e^{-\beta(|\mu|+|\alpha|)}
$$

As the basis again consists of monomials, the proof follows that of Proposition IV.4.

At this point it becomes straightforward to evaluate the Chern character associated to $Q_{j}$ on general elements of $\mathcal{A}^{\text {pol }}$. However, as this essentially repeats the statement of Theorem IV.5, we will not write the result. We confine ourselves to writing the integral representation for the functional evaluated on Toeplitz operators.

Theorem V.8. Let $f_{0}, \ldots, f_{2 k} \in C^{\infty}(V)$ have bounded first derivatives. On $C^{\infty}(\mathcal{M})$ define the differential operator

$$
\theta \cdot \frac{\partial}{\partial z}:=\sum_{i} \theta_{i} \frac{\partial}{\partial z_{i}}
$$

Then, for $j=1,2$,

$$
\begin{aligned}
& \mathrm{Ch}_{2 k}^{\beta}\left(Q_{j}\right)\left(T_{r}\left(f_{0}\right), \ldots, T_{r}\left(f_{2 k}\right)\right) \\
& =(-1)^{(j-1) k} \beta^{-k} \int_{I_{2 k+1}^{\beta}} \int_{\mathcal{X}^{2 k+1}} \delta\left(\sum_{n=0}^{2 k} s_{n}-\beta\right) \prod_{l=0}^{2 k} K^{r}\left(e^{-s_{l}} Z_{l}, Z_{l+1}\right) \\
& \quad \times f_{0}\left(z_{0}\right) \prod_{m=1}^{2 k}\left\{\theta_{m} \cdot \frac{\partial}{\partial z_{m}} f_{m}\left(z_{m}\right)+(-1)^{j} \bar{\theta}_{m} \cdot \frac{\partial}{\partial \bar{z}_{m}} f_{m}\left(z_{m}\right)\right\} \prod_{n=0}^{2 k} d \mu_{r}\left(Z_{n}\right) d^{2 k+1} s,
\end{aligned}
$$

where $Z_{2 k+1}:=Z_{0}$. 


\section{References}

1. Berezin, F.A.: General concept of quantization, Comm. Math. Phys. 40 (1975), 153174.

2. Berezin, F.A.: Introduction to Superanalysis, D. Reidel Publ. Co., Dordrecht (1987).

3. Born, M., and Jordan, P.: Zur Quantenmechanik, Z. Physik, 34 (1925), 858-888.

4. Born, M., Heisenberg, W., and Jordan, P.: Zur Quantenmechanik II, Z. Physik, 35 (1926), 557-615.

5. Borthwick, D., Klimek, S., Lesniewski, A., and Rinaldi, M.: Super Toeplitz operators and non-perturbative deformation quantization of supermanifolds, Comm. Math. Phys., 153 (1993), 49-76.

6. Borthwick, D., Klimek, S., Lesniewski, A., and Rinaldi, M.: Cartan superdomains, Super Toeplitz operators and deformation quantization, to appear.

7. Borthwick, D., Lesniewski, A., and Upmeier, H.: Non-perturbative deformation quantization of Cartan domains, J. Funct. Anal., 113 (1993), 153-176.

8. Coburn, L.A.: Deformation estimates for the Berezin-Toeplitz quantization, Comm. Math. Phys., 149 (1992), 415-424.

9. Connes, A.: Non-commutative differential geometry, Publ. Math. IHES, 62 (1986), 94-144.

10. Connes, A.: Entire cyclic cohomology of Banach algebras and characters of $\theta$-summable Fredholm modules, K-Theory, 1 (1988), 519-548.

11. Connes, A. and Moscovici, H.: Transgression and the Chern character in non-commutative K-homology, Comm. Math. Phys., to appear.

12. Dirac, P.A.M.: The fundamental equations of quantum mechanics, Proc. Roy. Soc., A109 (1926), 642-653.

13. Ernst, K., Feng, P., Jaffe, A., and Lesniewski, A.: Quantum K-theory, II. Homotopy invariance of the Chern character, J. Funct. Anal., 90 (1990), 355-368.

14. Getzler, E., and Szenes, A.: On the Chern character of a theta-summable Fredholm module, J. Funct. Anal., 84 (1989), 343-357.

15. Heisenberg, W.: Über quantentheoretische Umdeutung kinematischer und mechanischer Beziehungen, Z. Physik, 33 (1925), 879-893.

16. Jaffe, A., Lesniewski, A., and Osterwalder, K.: Quantum K-theory, I. The Chern character, Comm. Math. Phys., 118 (1988), 1-14.

17. Klimek, S., and Lesniewski, A.: Quantum Riemann surfaces, I. The unit disc, Comm. Math. Phys., 146 (1992), 103-122.

18. Reed, M., and Simon, B.: Methods of Modern Mathematical Physics, II: Fourier Analysis, Self-Adjointness, Academic Press, New York, San Francisco, London (1975).

19. Rieffel, M. A.: Deformation quantization of Heisenberg manifolds, Comm. Math. Phys., 122 (1989), 531-562.

20. Rieffel, M. A.: Non-commutative tori - a case study of non-commutative differentiable manifolds, Cont. Math., 105 (1990), 191-211.

21. Witten, E.: Constraints on supersymmetry breaking, Nucl. Phys., B202 (1982), 253316. 